\title{
Outcome of expectantly managed small-for-gestational-age pregnancies with normal Doppler parameters- a prospective cohort study
}

\author{
Sneha Mishra, Krishna Agarwal*
}

Department of Obstetrics and Gynecology, Maulana Azad Medical College, New Delhi, India

Received: 16 January 2021

Accepted: 11 February 2021

\author{
*Correspondence: \\ Dr. Krishna Agarwal, \\ E-mail: drkrishna.agarwal@gmail.com
}

Copyright: () the author(s), publisher and licensee Medip Academy. This is an open-access article distributed under the terms of the Creative Commons Attribution Non-Commercial License, which permits unrestricted non-commercial use, distribution, and reproduction in any medium, provided the original work is properly cited.

\begin{abstract}
Background: SGA foetuses with normal Dopplers are not at risk of IUD. However, there is lack of consensus about timing of delivery of SGA foetuses. Clinicians commonly induce all SGA pregnancies at 37 weeks. Expectant management of SGA foetuses beyond 37 weeks is not well studied.

Methods: We followed up women with clinically suspected growth restriction with foetal biometry, Doppler and biophysical profile. Pregnancies with foetal AC between $10^{\text {th }}$ to $3^{\text {rd }}$ centile with normal Dopplers were recruited in the study group. The women were allowed to go in spontaneous labor till 39+6/7 weeks or were induced at 39+6/7 weeks. The outcome of such cases was compared with controls who were induced at $37+0 / 7$ weeks.

Results: Spontaneous labor occurred in $42 \%$ subjects in study group whereas in control group all were induced. Mean gestation at delivery in the study group was increased (39.57 \pm 0.71 versus $37.0 \pm 0.0, \mathrm{p}$ value $<0.001)$. Almost $81 \%$ of the subjects in study group delivered after 39 weeks. The rate of caesarean section was significantly lower in study group (3\% versus $22 \%$, p value-0.024). Also, the risk of intrapartum foetal distress was lower in study group (3/36 versus 1/36). The mean birth weight in the study group was higher $(2426.5 \pm 154.1$ gm versus $2297.9 \pm 101.4 \mathrm{gm}, \mathrm{p}$ value $<0.001$.

Conclusions: Expectant management of SGA pregnancies with normal Doppler parameters leads to a significant increase in gestational age at birth and the mean birth weight and a significant reduction in caesarean section rate.
\end{abstract}

Keywords: Expectant management, Induction of labor, Mean gestation, SGA, Spontaneous labor

\section{INTRODUCTION}

A fetus is defined as small for gestational age (SGA) when the fetal abdominal circumference (AC) or estimated fetal weight $(\mathrm{EFW})$ is less than $10^{\text {th }}$ centile for the gestational age. $70 \%$ of the SGA fetuses are constitutionally small and do not have any risk while $30 \%$ are pathologically small and are at risk of various complications or have milder form of fetal growth restriction (FGR) with normal Doppler parameters. ${ }^{1}$ A Fetus with growth restriction is at increased risk of perinatal morbidity and mortality and poor long term health outcomes, such as impaired neurological and cognitive development and cardiovascular and endocrine diseases in adulthood. ${ }^{1}$ The healthy SGA fetuses as compared to the growth restricted fetuses do not suffer from such adverse perinatal outcomes. There are no welldefined criteria to differentiate between healthy SGA fetus and fetus with growth restriction, therefore all SGA fetuses are treated as FGR.

Some investigators have proposed criteria for distinguishing between the two and accordingly an SGA fetus is labeled as FGR when it is associated with any of the following. ${ }^{1,2}$

Abnormal umbilical artery (UA), middle cerebral artery (MCA) and uterine artery (Ut) doppler parameters, EFW or $\mathrm{AC}<3^{\text {rd }}$ centile.

There is lack of consensus about timing for delivery of healthy SGA and FGR fetuses. Clinicians commonly 
induce all SGA pregnancies even with normal Doppler at 37 weeks leading to higher rate of operative interventions and iatrogenic early birth. Induction of labor before 39 weeks is associated with increased cesarean section rate and neonatal respiratory distress. The timing of delivery is crucial in SGA pregnancies, and the risks of intrauterine fetal death versus the hazards of preterm delivery must be considered. Healthy and small SGA pregnancies are not at risk of intrauterine fetal death .Therefore, delivering these fetuses near term would be beneficial in terms of reducing need for labor induction and consequently, the cesarean section rate.

There are only few studies from the low and middle income countries on this subject and expectant management in SGA fetuses beyond 37 weeks is not very well studied. Hence our study provides new insight about the management of SGA fetuses. This study has special relevance in Indian settings with limited number of resources.

\section{METHODS}

The study was conducted in a tertiary care centre at New Delhi. Patients visiting antenatal clinic and admitted in maternity wards and labor room were included in the study from November 2017-March 2019 in this prospective cohort study.

Pregnancy suspected to be small, growth lagging behind by 3 weeks or more on abdominal palpation, were subjected to USG examination. Those who have $\mathrm{AC}<10^{\text {th }}$ centile and $>3^{\text {rd }}$ centile by intergrowth- 21 charts were enrolled as study subjects.

Women admitted in labor ward as a case of SGA with normal Doppler and being induced at or before 37 weeks of pregnancy were enrolled in control group.

\section{Inclusion criteria}

Reliable last menstrual period (LMP), POG 28-34 weeks and $\mathrm{AC}$ less than $10^{\text {th }}$ centile but more than $3^{\text {rd }}$ centile on intergrowth charts.

\section{Exclusion criteria}

Any medical disorders like known diabetes mellitus, hypertensive disorder, heart disease, respiratory disease, epilepsy, gross fetal congenital malformation on USG and Previous cesarean section.

\section{Statistical tests}

The quantitative variables in both groups were expressed as mean $\pm \mathrm{SD}$ and compared using unpaired t-test between groups and paired t-test within each group at various follow-ups. The qualitative variables were expressed as frequencies/percentages and compared using Chi-square test. A p value $<0.05$ was considered statistically significant. Statistical Package for Social sciences (SPSS) version 16.0 was used for statistical analysis.

\section{Ethical approval}

The study was approved by institutional ethics committee, Maulana Azad Medical College, New Delhi, India (F.No.17/IEC/MAMC/2017/OBG/14) on 27 October 2017

After taking written informed consent, the women were enrolled in study group and were followed up with DFMC, USG and Doppler studies. When the above parameters were in normal range, the pregnancy was allowed to continue till $396 / 7$ weeks and in case of any derangement in any of the above parameters, closer monitoring and induction of labor was done.

Women admitted in labor room with diagnosis of FGR fulfilling exclusion and inclusion criteria and were being induced at or before 37 weeks were enrolled in control group.

If all of the above parameters were normal, pregnancy was followed till $396 / 7$ weeks. If any monitoring parameter was deranged then close monitoring and delivery was undertaken. Umblical artery $\mathrm{PI}<95^{\text {th }}$, middle cerebral artery $\mathrm{PI}>5^{\text {th }}$ centile and uterine artery $\mathrm{PI}<95^{\text {th }}$ was considered as normal.

36 women were enrolled in each group. Approval from the institutional research ethics committee was taken.

\section{RESULTS}

A total of 82 women with pregnancy at 28 to 34 weeks of gestation who attended antenatal clinic were assessed for eligibility. Of 82 women, 46 women did not meet eligibility criteria and 36 women who met all the eligibility criteria were enrolled in the study group.

Table 1: Demographic and clinical details by groups.

\begin{tabular}{|c|c|c|c|}
\hline & $\begin{array}{l}\text { Study } \\
\text { group } \\
(n=36)\end{array}$ & $\begin{array}{l}\text { Control } \\
\text { group } \\
(n=36)\end{array}$ & $P$ value \\
\hline Age (years) & $23.69 \pm 2.59$ & $3.33 \pm 2.32$ & 0.535 \\
\hline \multicolumn{3}{|c|}{ Socioeconomic class } & \multirow{6}{*}{0.009} \\
\hline Lower & $2(5.6 \%)$ & $5(13.9 \%)$ & \\
\hline Upper lower & $10(27.8 \%)$ & $16(44.4 \%)$ & \\
\hline Lower middle & $24(66.7 \%)$ & $10(27.8 \%)$ & \\
\hline Upper middle & 0 & $3(8.3 \%)$ & \\
\hline Upper & 0 & $2(5.6 \%)$ & \\
\hline \multicolumn{3}{|l|}{ Parity } & \multirow{5}{*}{0.772} \\
\hline Primigravida & $17(47 \%)$ & $17(47 \%)$ & \\
\hline $2^{\text {nd }}$ gravid & $9(25 \%)$ & $12(33.3 \%)$ & \\
\hline $3^{\text {rd }}$ gravid & $8(22.2 \%)$ & $5(13.8 \%)$ & \\
\hline$>3^{\text {rd }}$ gravid & $2(5.5 \%)$ & $2(5.5 \%)$ & \\
\hline
\end{tabular}

Data expressed as Mean \pm SD and percentage (\%). 
For enrolment of subjects under the control group, a total of 110 women who were being induced in labor room for SGA pregnancies with normal Doppler parameters at 37 weeks were assessed for eligibility. Of 110 women, 74 women did not fulfil the eligibility criteria and 36 women who met all the eligibility criteria were enrolled in the control group.

The mean age of participants was comparable in both the groups. Most of the subjects in the study group belonged to lower middle socioeconomic status while most of the subjects in control group belonged to upper lower socioeconomic status by modified Kuppuswamy scale. In both the groups almost half of the subjects were primigravida.

The mean body mass index and hemoglobin was slightly higher in study group as compared to the control group. Subjects of both the groups were normotensive and the mean blood pressure in both the groups was comparable.

Table 2: Labor and neonatal details by groups.

\begin{tabular}{|c|c|c|c|}
\hline & Study group $(n=36)$ & Control group $(n=36)$ & P value \\
\hline \multicolumn{3}{|l|}{ Onset of labor } & \multirow{3}{*}{$<0.001$} \\
\hline Spontaneous & $15(42 \%)$ & 0 & \\
\hline Induced & $21(58 \%)$ & $36(100 \%)$ & \\
\hline Duration of first stage (hours) & $8.49 \pm 1.63$ & $8.88 \pm 1.24$ & 0.305 \\
\hline Duration of second stage (minutes) & $28.9 \pm 11.9$ & $29.6 \pm 8.9$ & 0.773 \\
\hline \multicolumn{3}{|l|}{ Mode of delivery } & \multirow{3}{*}{0.024} \\
\hline Vaginal & $35(97 \%)$ & $28(78 \%)$ & \\
\hline Cesarean & $1(3 \%)$ & $8(22 \%)$ & \\
\hline \multicolumn{3}{|l|}{ Mode of induction } & \multirow{4}{*}{$<0.001$} \\
\hline Single dose of cerviprime gel & $7(33.3 \%)$ & $4(12 \%)$ & \\
\hline Two doses of cerviprime gel & $8(38.0 \%)$ & $16(44 \%)$ & \\
\hline Two doses of cerviprime gel followed by oxytocin drip & $6(28.57 \%)$ & $16(44 \%)$ & \\
\hline Mean gestation at delivery (weeks) & $39.57 \pm 0.71$ & $37.0 \pm 0.0$ & $<0.001$ \\
\hline \multicolumn{3}{|l|}{ Timing of delivery } & \multirow{3}{*}{$<0.001$} \\
\hline$<39$ weeks period of gestation & $7(19 \%)$ & $36(100 \%)$ & \\
\hline$\geq 39$ weeks period of gestation & $29(81 \%)$ & 0 & \\
\hline Mean birth weight (grams) & $2426.5 \pm 154.1$ & $2297.9 \pm 101.4$ & $<0.001$ \\
\hline $\operatorname{APGAR}(\leq 7$ at 1 minutes $)$ & 0 & $1(3 \%)$ & 1.000 \\
\hline $\operatorname{APGAR}(\leq 7$ at 5 minutes $)$ & 0 & $1(3 \%)$ & 1.000 \\
\hline Need for ICU admission & 0 & $1(3 \%)$ & 1.000 \\
\hline Successful breastfeeding & $36(100 \%)$ & $35(97 \%)$ & 1.000 \\
\hline
\end{tabular}

In the study group $15(42 \%)$ subjects had spontaneous labor at or before $39+6$ weeks while rest $21(58 \%)$ were induced at $39+6$ weeks. The duration of first and second stage of labor was slightly more in control group as compared to study group. However, the difference was statistically insignificant. There was statistically significant difference between the groups with regard to the mode of delivery. Almost all the participants in the study group delivered vaginally. In the control group 8 (22\%) participants underwent cesarean section whereas in the study group only $1(3 \%)$ participant underwent cesarean section.

Labour induction required less number of doses of cerviprime gel in the study group as compared to the control group and less number of subjects needed oxytocin drip as compared to control group.

The incidence of intrapartum fetal distress and arrest of cervical dilatation was more among the control group. 3 subjects $(8.3 \%)$ in the control group developed intrapartum fetal distress whereas only 1 subject $(2.7 \%)$ in the study group developed it.

In the study group, no subject developed PPH, retained placenta, puerperal sepsis or required blood transfusion. In the control group, one participant had abruption of placenta and required one unit blood transfusion. Rest of the subjects did not develop any complication. The mean gestation at delivery in study group was significantly higher than control group.

It was observed that almost $81 \%$ of the subjects in the study group were able to deliver at or after 39 weeks of period of gestation whereas all the subjects in control group were induced at 37 weeks. The difference in the number of subjects delivering at or beyond 39 weeks of gestation was significantly higher in the study group as compared to the control group. The mean birth weight in the study group was higher as compared to control group 
and the difference was statically significant. However, APGAR score, need for NICU admission and successful breastfeeding at 72 hours were comparable between the two groups. On detailed analysis of the gestation at delivery, it was found that most of the subjects $(80.5 \%)$ delivered at $39-39+6$ weeks, $11.1 \%$ delivered at $38-38+6$ weeks and only $8.3 \%$ delivered at $37-37+6$ weeks.

\section{DISCUSSION}

Our study shows a significant increase in the mean gestational age at delivery with expectant management (39.57 \pm 0.71 weeks versus $37.0 \pm 0.0$ weeks). This is in concordance with the results of other studies. ${ }^{3-5}$ As many as $81 \%$ of the subjects in study group delivered after 39 weeks of gestation. Expectant management of SGA pregnancies is associated with more chances of going into spontaneous labour. The induction rate was $58 \%$ in study group compared to $100 \%$ of the control group. The expectantly managed group had much less induction time than the control group and they required less oxytocin ( $28 \%$ versus $44 \%$ ). The duration of $1^{\text {st }}$ stage of labor was less in study group than the control group $(8.49 \pm 1.63$ hours versus $8.88 \pm 1.24$ hours) though the difference was not statistically significant. The duration of second stage was comparable in both the groups.

The rate of cesarean section was lower in study group (3\% versus $22 \%$ ). Also, the risk of intrapartum fetal distress was higher in control group than study group $(3 / 36$ versus $1 / 36)$. The mean birth weight in the study group was higher than control group $(2426.5 \pm 154.1 \mathrm{gm}$ versus $2297.9 \pm 101.4 \mathrm{gm})$. However, the other perinatal outcomes were comparable.

There is paucity of studies that have elucidated the optimal timing of delivery in SGA pregnancies. There are only few studies on this subject and expectant management in SGA fetuses beyond 37 weeks is not very well studied. Hence this study provides new insight about the management of SGA fetuses.

Our study proves that the pregnancy having fetus with $\mathrm{AC}$ between $3^{\text {rd }}$ and $10^{\text {th }}$ centile with normal fetal Doppler parameters and normal biophysical profile can be safely managed expectantly till $39+6 / 7$ weeks of gestation.

The main strength of this study is that SGA fetuses with $\mathrm{AC}$ between $3^{\text {rd }}$ to $10^{\text {th }}$ centile were included. In all other studies, the study subjects were SGA pregnancies with $\mathrm{AC}$ or EFW $<10^{\text {th }}$ centile.

The DIGITAT trail which was a randomized control trial about induction versus expectant management for intrauterine growth restricted babies at term showed no difference in adverse outcomes between induction and expectant management until 40 weeks of gestation. ${ }^{6}$ ACOG also recommends delivery between 38-39 6/7 weeks in SGA fetuses with normal dopplers. ${ }^{7}$

Our study also shows similar results and further research should be done in low and middle income countries to determine the optimal timing of delivery for SGA fetuses.

\section{CONCLUSION}

Expectant management of small-for-gestational-age pregnancies with normal Doppler parameters leads to significant increase in mean gestation at delivery and mean birth weight and a significant decrease in cesarean section rates.

Funding: No funding sources

Conflict of interest: None declared

Ethical approval: The study was approved by the Institutional Ethics Committee Maulana Azad Medical College, New Delhi, India (F.No.17/IEC/MAMC/2017/OBG/14) on 27/10/2017

\section{REFERENCES}

1. Figueras F, Gratacos E. An integrated approach to fetal growth restriction. Best Pract Res Clin Obstet Gynaecol. 2017;38:48-58.

2. Lausman A, Kingdom J, Gagnon R, Basso M, Bos $\mathrm{H}$, Crane $\mathrm{J}$, et al. Intrauterine growth restriction: screening, diagnosis and management. J Obstet Gynaecol Can. 2013;35:741-57.

3. Veglia M, Cavallaro A, Papageorghiou A, Black R, Impey L. Small-for-gestational-age babies after 37 weeks: impact study of risk-stratification protocol. Ultrasound Obstet Gynecol. 2018;52:66-71.

4. Hidaka N, Sato Y, Kido S, Fujita Y, Kato K. Expectant management of pregnancies complicated by fetal growth restriction without any evidence of placental dysfunction at term: Comparison with routine labor induction. J Obstet Gynaecol Res. 2018;44:93-101.

5. Boers KE, van der Post JA, Mol BW, van Lith JM, Scherjon SA. Labour and neonatal outcome in small for gestational age babies delivered beyond $36+0$ weeks: a retrospective cohort study. J Pregnanc. 2011;2011:293516.

6. Boers KE, Vijgen SM, Bijlenga D, van der Post JA, Bekedam DJ, Kwee A, et al. Induction versus expectant monitoring for intrauterine growth restriction at term: randomised equivalence trial (DIGITAT). BMJ. 2010;341:c7087.

7. American College of Obstetricians and Gynecologists. ACOG Practice bulletin no. 134: fetal

Cite this article as: Mishra S, Agarwal K. Outcome of expectantly managed small-for-gestational-age pregnancies with normal Doppler parameters- a prospective cohort study. Int J Reprod Contracept Obstet Gynecol 2021;10:1134-7. 